\title{
A Perovskite-Like Framework Encapsulated with Fe-based Magnetic Units in $\mathrm{Ba}_{10} \mathrm{Fe}_{3} \mathrm{Sb}_{7} \mathrm{Se}_{24}$
}

Wei-Cheng Lin ${ }^{\dagger}$, Hongsun $\mathrm{Ryu}^{\delta}$, Chen-I Yang*,*, Chien-Yen Weng ${ }^{\dagger}$, Joon Ik Jang ${ }^{\delta, *}$, and Kuei-Fang $\mathrm{Hsu}^{\dagger}, *$

$\dagger$ Department of Chemistry, National Cheng Kung University, Tainan 70101, Taiwan

\$Department of Chemistry, Tunghai University, Taichung 40704, Taiwan

${ }^{\delta}$ Department of Physics, Sogang University, 35 Baekbeom-ro, Mapo-gu, Seoul, 04107

South Korea

\section{Supplementary Data}

Figure. S1 SEM images. energy dispersive spectra and EDS results of $\mathrm{Ba}_{10} \mathrm{Fe}_{3} \mathrm{Sb}_{7} \mathrm{Se}_{24}$ on three single crystals.

Figure S2 Comparative PXRD of $\mathrm{Ba}_{10} \mathrm{Fe}_{3} \mathrm{Sb}_{7} \mathrm{Se}_{24}$ between the measured (black) and the calculated patterns (blue).

Figure $\mathbf{S 3}$ The layer parallel to the $a b$ plane fused together along the $c$ axis.

Figure S4 The $\mathrm{Fe}_{2} \mathrm{Se}_{6}$ dimers and $\mathrm{FeSe}_{4}$ tetrahedra trapped within and between the slabs viewed along the $c$ axis.

Figure S5 $\chi_{\mathrm{M}}{ }^{-1}(\circ)$ vs. $T$ plot of $\mathrm{Ba}_{10} \mathrm{Fe}_{3} \mathrm{Sb}_{7} \mathrm{Se}_{24}$. The red line represents the best fit to $\chi_{\mathrm{M}}{ }^{-1}$ above $30 \mathrm{~K}$ using the Curie-Weiss law.

Figure S6 $\chi_{\mathrm{M}} T$ vs. $T$ plot in the range of $2-300 \mathrm{~K}$ for $\mathrm{Ba}_{10} \mathrm{Fe}_{3} \mathrm{Sb}_{7} \mathrm{Se}_{24}$.

Figure S7 Tauc's plot of UV-vis-NIR optical absorption spectrum for $\mathrm{Ba}_{10} \mathrm{Fe}_{3} \mathrm{Sb}_{7} \mathrm{Se}_{24}$.

Table S1 Crystal data for $\mathrm{Ba}_{10} \mathrm{Fe}_{3} \mathrm{Sb}_{7} \mathrm{Se}_{24}$ collected at $200 \mathrm{~K}$ and $300 \mathrm{~K}$.

Table S2 Selected bond bistances $(\AA)$ for $\mathrm{Ba}_{10} \mathrm{Fe}_{3} \mathrm{Sb}_{7} \mathrm{Se}_{24}$ collected at $200 \mathrm{~K}$ and 300 K. 
Figure. S1 SEM images. energy dispersive spectra and EDS results of $\mathrm{Ba}_{10} \mathrm{Fe}_{3} \mathrm{Sb}_{7} \mathrm{Se}_{24}$ on three single crystals.
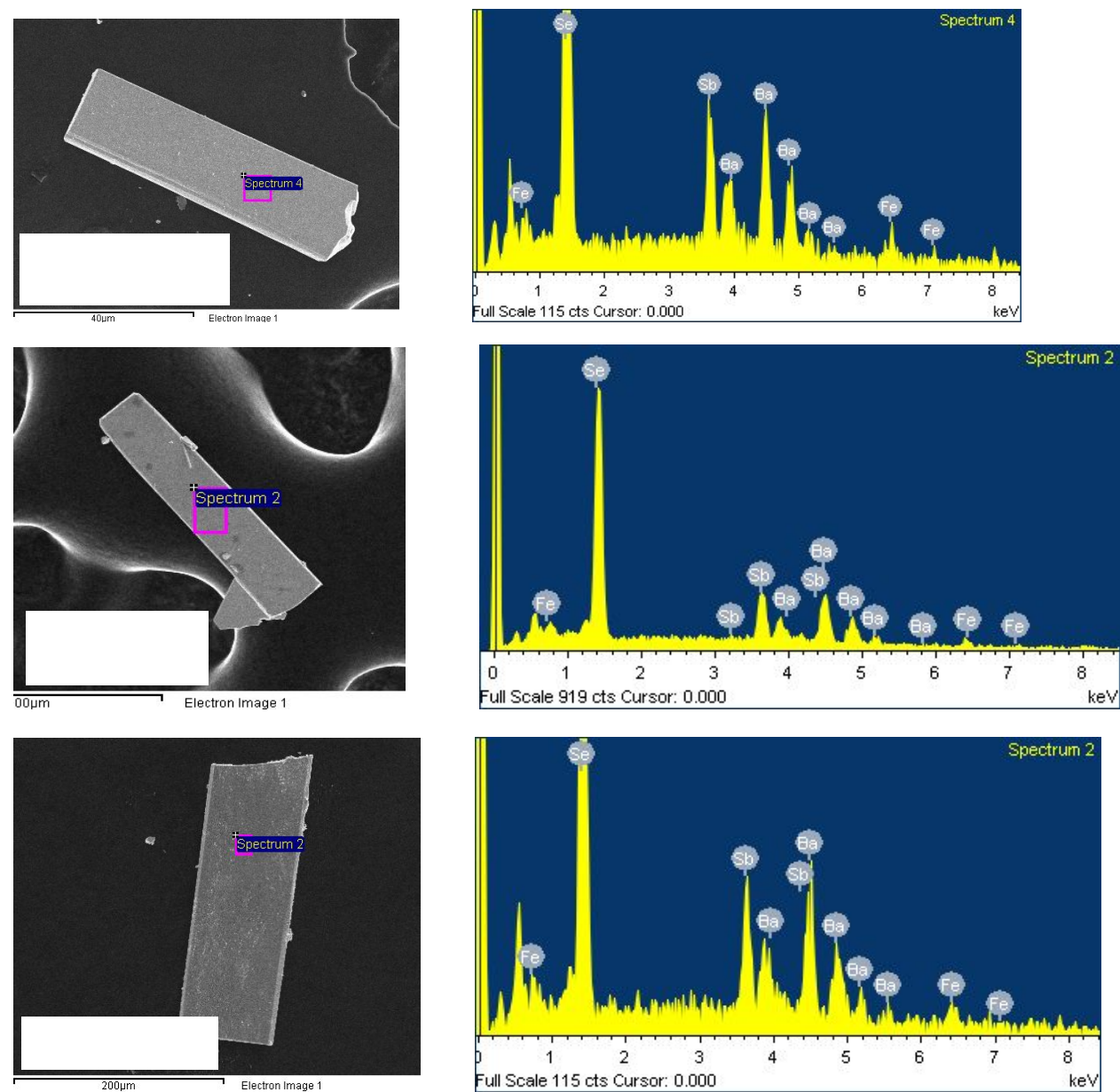

\begin{tabular}{|c|c|c|c|c|c|}
\hline & $\mathrm{Ba}$ & $\mathrm{Fe}$ & $\mathrm{Sb}$ & $\mathrm{Se}$ & Atomic Ratio \\
\hline Crystal 1 & 22.27 & 6.87 & 16.09 & 54.84 & $9.72: 3: 7.03: 23.95$ \\
\hline Crystal 2 & 22.34 & 6.82 & 16.98 & 53.87 & $9.83: 3: 7.47: 23.70$ \\
\hline Crystal 3 & 22.35 & 6.85 & 16.23 & 54.57 & $9.79: 3: 7.11: 23.90$ \\
\hline
\end{tabular}


Figure S2 Comparative PXRD of $\mathrm{Ba}_{10} \mathrm{Fe}_{3} \mathrm{Sb}_{7} \mathrm{Se}_{24}$ between the measured (black) and the calculated patterns (blue).

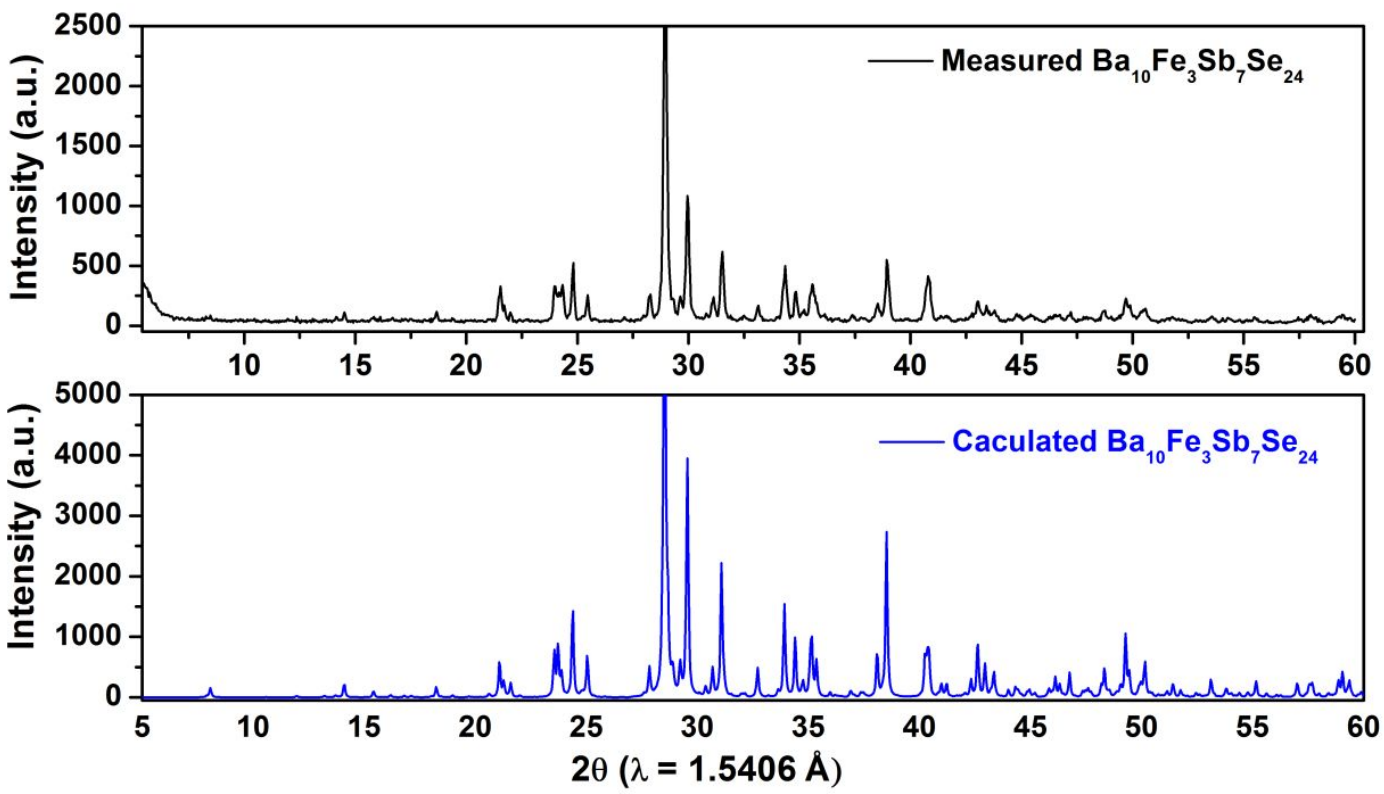


Figure S3 A perspective along the $a$ axis to show the layer parallel to the $a b$ plane fused together.

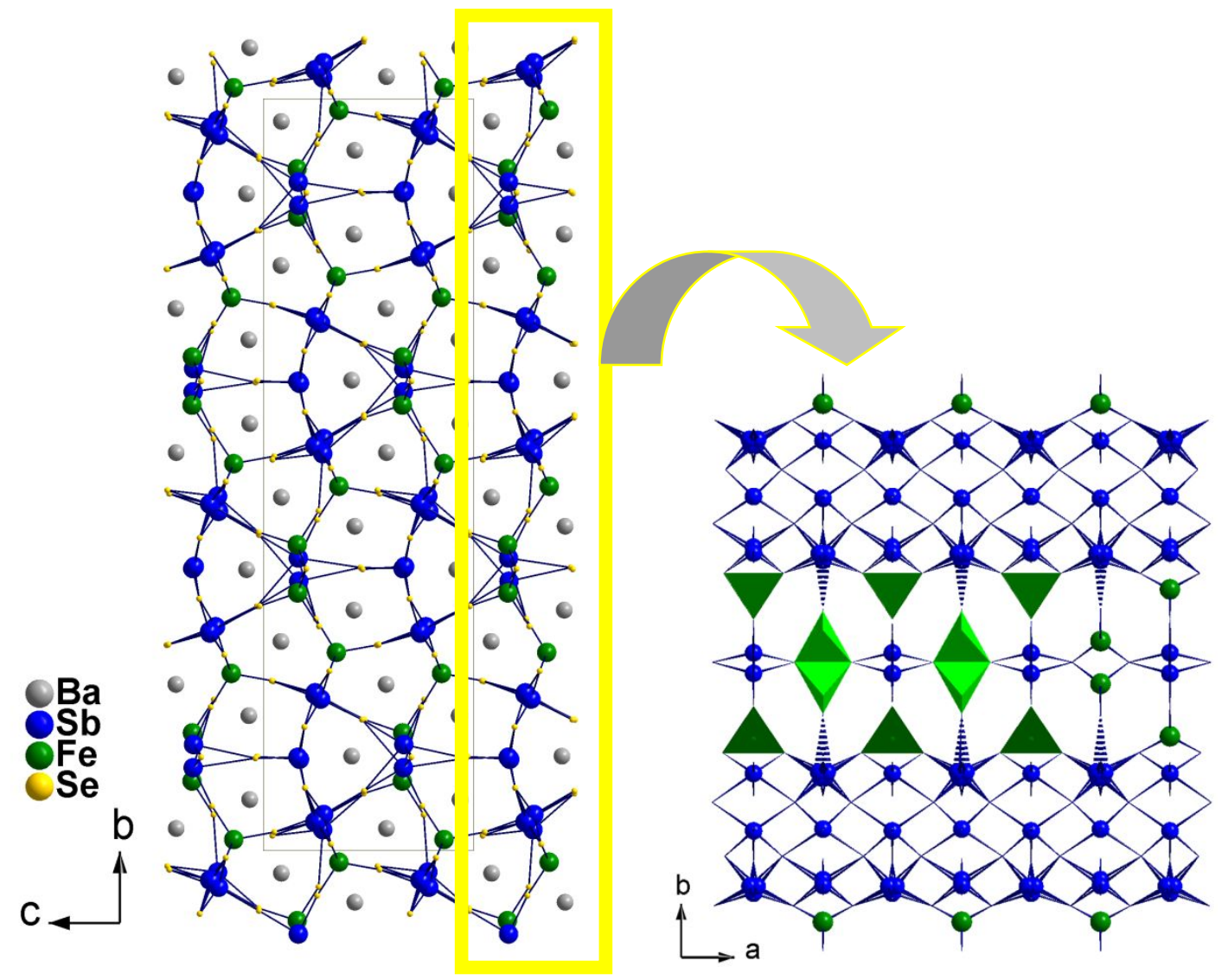


Figure $\mathbf{S} 4$ The $\mathrm{Fe}_{2} \mathrm{Se}_{6}$ dimers and $\mathrm{FeSe}_{4}$ tetrahedra trapped within and between the slabs viewed along the $c$ axis.

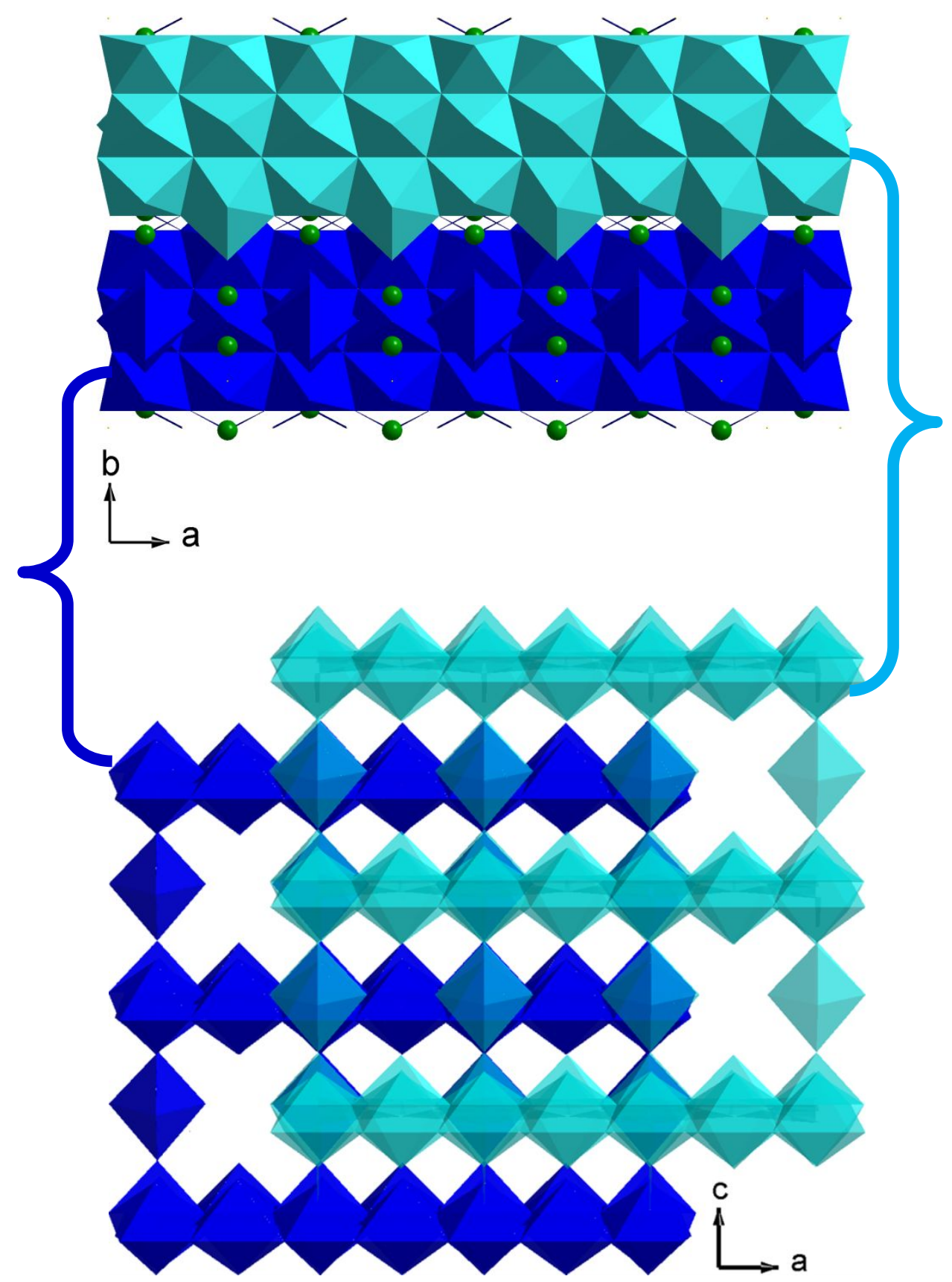


Figure S5 $\chi_{\mathrm{M}}{ }^{-1}(\circ)$ vs. $T$ plot of $\mathrm{Ba}_{10} \mathrm{Fe}_{3} \mathrm{Sb}_{7} \mathrm{Se}_{24}$. The red line represents the best fit to $\chi_{\mathrm{M}}^{-1}$ above $30 \mathrm{~K}$ using the Curie-Weiss law.

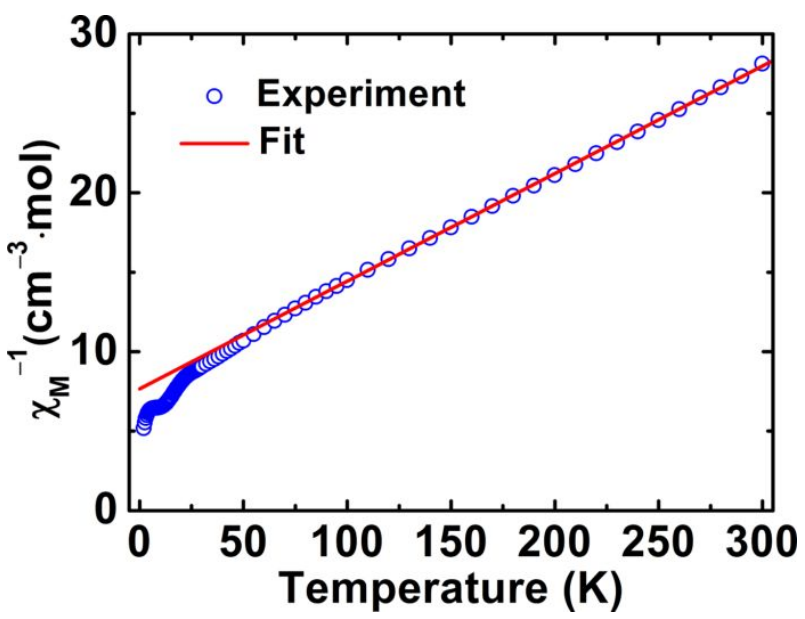

Figure $\mathbf{S 6} \chi_{\mathrm{M}} T$ vs. $T$ plot in the range of $2-300 \mathrm{~K}$ for $\mathrm{Ba}_{10} \mathrm{Fe}_{3} \mathrm{Sb}_{7} \mathrm{Se}_{24}$.

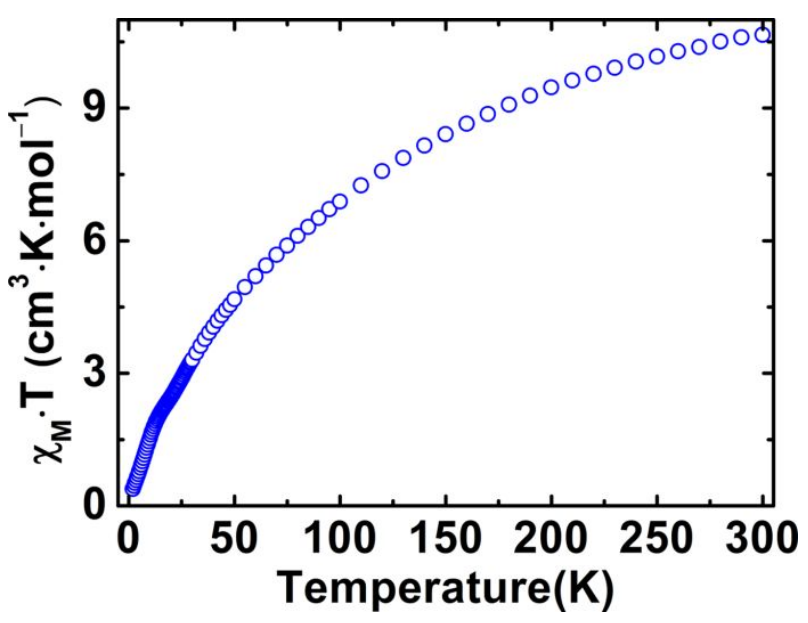


Figure S7 Tauc's plot of UV-vis-NIR optical absorption spectrum for $\mathrm{Ba}_{10} \mathrm{Fe}_{3} \mathrm{Sb}_{7} \mathrm{Se}_{24}$.

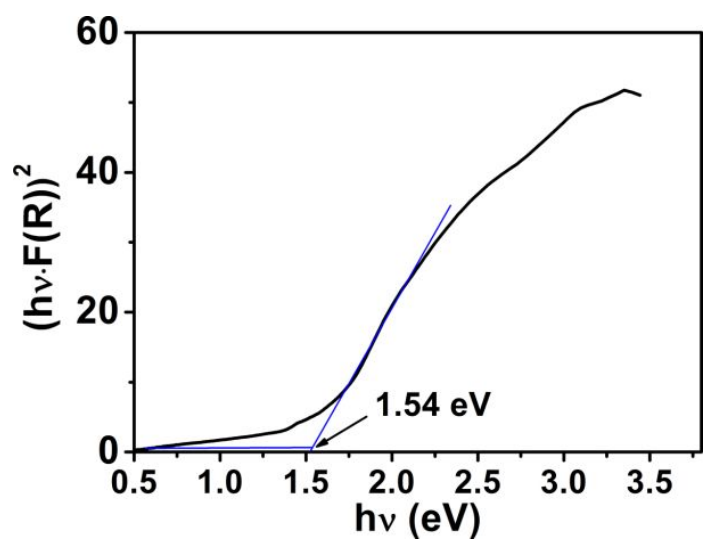


Table S1 Crystal data for $\mathrm{Ba}_{10} \mathrm{Fe}_{3} \mathrm{Sb}_{7} \mathrm{Se}_{24}$ collected at $200 \mathrm{~K}$ and $300 \mathrm{~K}$.

\begin{tabular}{|c|c|c|}
\hline Temperature & $200 \mathrm{~K}$ & $300 \mathrm{~K}$ \\
\hline Wavelength $(\AA)$ & 0.71073 & 0.71073 \\
\hline Crystal system & Orthorhombic & Orthorhombic \\
\hline Space group & $\mathrm{Cmc} 2_{1}$ & $\mathrm{Cmc}_{1}$ \\
\hline$a(\AA)$ & $9.3310(9)$ & $9.3406(2)$ \\
\hline$b(\AA)$ & $44.5262(3)$ & $44.6052(10)$ \\
\hline$c(\AA)$ & $12.5343(9)$ & $12.5528(2)$ \\
\hline$V\left(\AA^{3}\right)$ & $5207.7(6)$ & $5229.99(18)$ \\
\hline$Z$ & 4 & 4 \\
\hline$\rho_{\text {calcd }}\left(\mathrm{g} / \mathrm{cm}^{3}\right)$ & 5.469 & 5.446 \\
\hline Absorption coefficient $\left(\mathrm{mm}^{-1}\right)$ & 28.569 & 28.447 \\
\hline $\mathrm{F}(000)$ & 7244.0 & 7244 \\
\hline Crystal size $\left(\mathrm{mm}^{3}\right)$ & $0.440 \times_{0.076} \times_{0.032}$ & $0.440 \times 0.076 \times 0.032$ \\
\hline Theta range for data collection & 1.564 to $28.376^{\circ}$ & 1.564 to $28.376^{\circ}$ \\
\hline Reflections collected & 40233 & 41027 \\
\hline Independent reflections & $4942[\mathrm{R}(\mathrm{int})=0.0647]$ & $4919[\mathrm{R}(\mathrm{int})=0.0613]$ \\
\hline Goodness-of-fit on $\mathrm{F}^{2}$ & 1.039 & 1.063 \\
\hline Final R indices [I $>2 \operatorname{sigma}(\mathrm{I})]$ & ${ }^{a} R_{1}=0.0468,{ }^{b} R_{2}=0.1493$ & ${ }^{a} R_{1}=0.0465,{ }^{b} \mathrm{w} R_{2}=0.1445$ \\
\hline R indices (all data) & ${ }^{a} R_{1}=0.0605,{ }^{b} R_{2}=0.1497$ & ${ }^{a} R_{1}=0.0543,{ }^{b} \mathrm{w} R_{2}=0.1445$ \\
\hline Largest diff. peak and hole $\left(\mathrm{e}^{\AA^{-3}}\right)$ & 1.83 and $-2.57 \mathrm{e}^{-3}$ & 2.25 and $-2.43 \mathrm{e}^{-3}$ \\
\hline
\end{tabular}


Table S2 Selected bond bistances $(\AA)$ for $\mathrm{Ba}_{10} \mathrm{Fe}_{3} \mathrm{Sb}_{7} \mathrm{Se}_{24}$ collected at $200 \mathrm{~K}$ and $300 \mathrm{~K}$.

\begin{tabular}{|c|c|c|}
\hline & $200 K$ & $300 \mathrm{~K}$ \\
\hline \multirow[t]{5}{*}{$\mathrm{Sb} 1$} & $2.542(4)$ & $2.541(4)$ \\
\hline & $2.611(3)$ & $2.613(2)$ \\
\hline & $2.611(3)$ & $2.613(2)$ \\
\hline & $3.376(3)$ & $3.379(2)$ \\
\hline & $3.376(3)$ & $3.379(2)$ \\
\hline \multirow[t]{5}{*}{$\mathrm{Sb} 2$} & $2.530(4)$ & $2.530(4)$ \\
\hline & $2.611(3)$ & $2.612(2)$ \\
\hline & $2.611(3)$ & $2.612(2)$ \\
\hline & $3.346(3)$ & $3.350(2)$ \\
\hline & $3.346(3)$ & $3.350(2)$ \\
\hline \multirow[t]{6}{*}{$\mathrm{Sb} 3$} & $2.594(4)$ & $2.593(4)$ \\
\hline & $2.665(3)$ & $2.659(3)$ \\
\hline & $2.665(3)$ & $2.660(3)$ \\
\hline & $3.326(4)$ & $3.334(4)$ \\
\hline & $3.457(3)$ & $3.461(2)$ \\
\hline & $3.457(3)$ & $3.461(2)$ \\
\hline \multirow[t]{6}{*}{$\mathrm{Sb} 4$} & $2.734(14)$ & $2.758(12)$ \\
\hline & $2.734(14)$ & $2.758(12)$ \\
\hline & $2.849(16)$ & $2.852(14)$ \\
\hline & $3.081(16)$ & $3.086(14)$ \\
\hline & $3.097(11)$ & $3.093(9)$ \\
\hline & $3.097(11)$ & $3.093(9)$ \\
\hline \multirow[t]{6}{*}{$\mathrm{Sb} 4 \mathrm{a}$} & $2.686(14)$ & $2.680(13)$ \\
\hline & $2.731(15)$ & $2.732(14)$ \\
\hline & $2.841(16)$ & $2.858(16)$ \\
\hline & $2.995(14)$ & $2.992(14)$ \\
\hline & $3.179(16)$ & $3.191(15)$ \\
\hline & $3.290(15)$ & $3.299(13)$ \\
\hline \multirow[t]{6}{*}{ Sb5 } & $2.615(6)$ & $2.606(5)$ \\
\hline & $2.615(6)$ & $2.606(5)$ \\
\hline & $2.619(9)$ & $2.623(7)$ \\
\hline & $3.296(9)$ & $3.303(8)$ \\
\hline & $3.419(5)$ & $3.427(4)$ \\
\hline & $3.419(5)$ & $3.427(4)$ \\
\hline Sb5a & $2.677(7)$ & $2.676(6)$ \\
\hline
\end{tabular}




\begin{tabular}{|c|c|c|}
\hline & $2.677(7)$ & $2.676(6)$ \\
\hline & $2.736(10)$ & $2.741(9)$ \\
\hline & $3.157(10)$ & $3.160(8)$ \\
\hline & $3.383(6)$ & $3.384(5)$ \\
\hline & $3.383(6)$ & $3.384(5)$ \\
\hline \multirow[t]{6}{*}{ Sb6 } & $2.590(6)$ & $2.592(4)$ \\
\hline & $2.794(6)$ & $2.805(3)$ \\
\hline & $2.794(6)$ & $2.805(3)$ \\
\hline & $3.072(5)$ & $3.074(3)$ \\
\hline & $3.072(5)$ & $3.074(3)$ \\
\hline & $3.375(5)$ & $3.386(4)$ \\
\hline \multirow[t]{7}{*}{ Sb6a } & $2.810(56)$ & $2.784(30)$ \\
\hline & $2.810(56)$ & $2.784(30)$ \\
\hline & $2.901(104)$ & $3.048(74)$ \\
\hline & $3.207(118)$ & 3.184(51) \\
\hline & $3.208(118)$ & $3.282(72)$ \\
\hline & $3.270(63)$ & $3.282(72)$ \\
\hline & $3.423(118)$ & $3.415(68)$ \\
\hline \multirow[t]{7}{*}{ Sb7 } & $2.555(6)$ & $2.547(5)$ \\
\hline & $2.785(6)$ & $2.791(6)$ \\
\hline & $2.864(3)$ & $2.869(2)$ \\
\hline & $2.864(3)$ & $2.869(2)$ \\
\hline & $3.635(7)$ & $3.652(6)$ \\
\hline & $3.757(6)$ & $3.767(5)$ \\
\hline & $3.766(6)$ & $3.768(5)$ \\
\hline \multirow[t]{7}{*}{ Sb7a } & $2.502(5)$ & $2.506(5)$ \\
\hline & $2.805(7)$ & $2.810(6)$ \\
\hline & $2.889(3)$ & $2.893(3)$ \\
\hline & $2.889(3)$ & $2.893(3)$ \\
\hline & $3.686(6)$ & $3.690(6)$ \\
\hline & $3.750(6)$ & $3.759(5)$ \\
\hline & $3.782(6)$ & $3.784(5)$ \\
\hline
\end{tabular}

